\title{
Optical-fiber thermal-wave-cavity technique to study thermal properties of silver/clay nanofluids
}

\author{
M. Noroozi \\ monir.noroozi@gmail.com
}

\section{S. Radiman}

\section{A. Zakaria}

\section{K. Shameli}

\section{Deraman}

\section{S. Soltaninejad}

\section{A. Abedini}

\author{
School of Applied Physics, Faculty of Science and Technology, Universiti Kebangsaan Malaysia, 43600 \\ UKM Bangi, Selangor, Malaysia \\ School of Applied Physics, Faculty of Science and Technology, Universiti Kebangsaan Malaysia, 43600 \\ UKM Bangi, Selangor, Malaysia \\ Department of Physics, Faculty of Science, Universiti Putra Malaysia, 43400 UPM Serdang, Selangor, \\ Malaysia \\ Department of Physics, Faculty of Science, Universiti Putra Malaysia, 43400 UPM Serdang, Selangor, \\ Malaysia \\ School of Applied Physics, Faculty of Science and Technology, Universiti Kebangsaan Malaysia, 43600 \\ UKM Bangi, Selangor, Malaysia \\ School of Applied Physics, Faculty of Science and Technology, Universiti Kebangsaan Malaysia, 43600 \\ UKM Bangi, Selangor, Malaysia \\ School of Applied Physics, Faculty of Science and Technology, Universiti Kebangsaan Malaysia, 43600 \\ UKM Bangi, Selangor, Malaysia
}

Thermal properties enhancement of nanofluids have varied strongly with synthesis technique, particle size and type, concentration and agglomeration with time. This study explores the possibility of changing the thermal wave signal of $\mathrm{Ag} / \mathrm{clay}$ nanofluids into a thermal diffusivity measurement at well dispersion or aggregation of nanoparticles in the base fluid. Optical-Fiber Thermal-Wave-Cavity (OF-TWC) technique was achieved by using a small amount of nanofluid (only $0.2 \mathrm{~mL}$ ) between fiber optic tip and the Pyroelectric detector and the cavity-length scan was performed. We established the accuracy and precision of this technique by comparing the thermal diffusivity of distilled water to values reported in the literature. Assuming a linear Pyroelectric signal response, the results show that adding clay reduced the thermal diffusivity of water, while increasing the Ag concentration from 1 to $5 \mathrm{wt}$.\% increased the thermal diffusivity of the Ag nanofluid from $1.524 \times 10^{-3}$ to $1.789 \times 10^{-3} \mathrm{~cm}^{2} / \mathrm{s}$. However, in particular, nanoparticles show the tendency to form aggregates over time that correlated with the performance change of thermal properties of nanofluid. Our results confirm the high sensitivity of OF-TWC technique raises the potential to be applied to measuring the optical and thermal properties of nanofluids. Furthermore, this technique allows the extraction of information not obtained using other traditional techniques.

[DOI: http://dx.doi.org/10.2971/jeos.2014.14046]

Keywords: Silver nanoparticles, nanofluids, photopyroelectric technique, thermal diffusivity

\section{INTRODUCTION}

Nanofluids are a new category of more efficient heat-transport fluids that show promise in many applications, including in medical therapies, heat generation, and heat transfer [1]. It is important to study nanofluids with metal nanoparticles (NPs), especially silver NPs (Ag NPs), to understand their extraordinary thermal properties and how the size, shape, and concentration of the NPs affect those properties [2]. The structure of the Ag/clay nanofluid reduces the probability of $\mathrm{Ag}$ particles colliding, preventing particle aggregation and improving long-term stability of the suspension [3,4], based on the crystalline structure and the interlayer spaces of montmorillonite (MMT) clay [5]. However, in particular, NPs show a much stronger aggregation tendency that strongly influence on the local NPs concentration as well as thermal properties of nanofluids [6]. Therefore, the effects of nanoparticle agglomeration over time is difficult to separate from thermal property data of most of nanofluids. Although thermal properties of nanofluids are intensely researched at present, studies on thermal conductivity are more common in the literature than those reporting on thermal diffusivity of nanofluids [7]-[10]. There is little thermal diffusivity measurement using thermal lens spectrometry and the transient double-hot-wire method $[11,12]$. However, these techniques are disadvantageous because they require high-volume samples and are expensive and their long measurement time, without according the effect of aggregation time. Additionally, the complexity of their underpinning theoretical models can still be reduced while maintaining accurate results. Finally, these methods often require high temperatures to obtain reasonable signal-to-noise (SNR) ratios, which can increase the sample temperature and thus the measurement error [13].

The photopyroelectric (PPE) technique, called thermal-wavecavity (TWC) technique, has been well established for non- 
destructive measurement of thermal diffusivity, by a sensitive pyroelectric sensor, in thermal contact with the sample [14]. This method can measure thermal diffusivity by analyzing the amplitude of the pyroelectric (PE) signal from a cavitylength scan or frequency scan. The major advantage of cavity length scan is fixed the modulated frequency, which improves the signal-to-noise ratio (SNR). In a previous work [15], we developed the optical-fiber-based TWC (OF-TWC) technique, which has many advantages over the traditional TWC technique, including its high sensitivity in PE signal, simple operation, and low cost; it is especially advantageous because of the smaller sample volume it requires, while, the measurement time can be significantly shorter [16].

In the present study, we synthesized Ag NPs in the interlayer spaces of MMT clay in an aqueous solution, and then used the OF-TWC technique to measure thermal diffusivity of the $\mathrm{Ag} /$ clay nanofluids as a function of the $\mathrm{AgNO}_{3}$ concentration. The major advantage of this configuration is that a simple and sensitive minimum volume PE cell is designed and fabricated for room temperature measurement. The possibility of changing the PE signal into a thermal diffusivity measurement of nanofluid over time and the effect of nanoparticle aggregation on thermophysical properties also was investigated. We chose the OF-TWC technique because of its simplicity, short measurement interval (5 $\mathrm{min}$ ), and the small volume of liquid required per measurement $(0.2 \mathrm{~mL})$, an essential feature when dealing with expensive or difficult-to-synthesize samples.

\section{BASIC PHOTOPYROELECTRIC THEORY}

The principle and procedures of the PPE method, named the OF-TWC technique, have been described elsewhere $[15,16]$. Matvienko and Mandelis (2006) [17] reduced the three-dimensional model of the OF-TWC technique to a onedimensional model by using a thermal wave generator with a larger diameter than that of the laser spot size. Considering a thermal-wave (TW) generator with a larger diameter than the thermal diffusion length of the sample, the temperature field of the sample can be represented by a one-dimensional model, and the PE signal detected at a fixed TW oscillation frequency $f=\omega / 2 \pi$ is given by [17]

$$
V\left(L, \alpha_{l}, \omega\right)=\operatorname{CONST}(\omega)\left[\frac{e^{-\sigma_{l} L}}{1-\gamma_{l s} \gamma_{p l} e^{2 \sigma_{l} L}}\right]
$$

where $\gamma$ is the TW reflection coefficient and $\sigma$ is the complex TW diffusion coefficient $\sigma_{j}=(1+i) / \mu_{j}$, and $\mu=(\alpha / \pi f)^{1 / 2}$ is the thermal diffusion length of sample at frequency $f$. The term Const $(\omega)$ in Eq. (1), is a complex expression containing parameters including the laser intensity and thermal properties of the substances involved (the PE generator, PE detector, and air surrounding the cavity); this term is independent of the cavity length. The subscripts $s, p$, and lrefer to the plane metallic light absorber (TW generator), PE detector material, and liquid sample, respectively. In a thermally thick sample, the thermal diffusion length is smaller than the thickness of the sample's thermally thick region $\left(\mu_{s}<L_{S}\right)$ [18]. For samples in the thermally thick condition, $e^{-2 \sigma_{i} l_{i}} \approx 0$, Eq. (1) can be written more simply as

$$
|V(f, L)|=C(f) \times e^{-L / \mu}
$$

where $V$ and $L$ are the amplitude of PE signal and the cavity length, respectively, and $\alpha$ is the thermal diffusivity of the liquid sample. The slope fitting parameter $A=(\pi f / \alpha)^{1 / 2}$ of a linear equation in a semilog scale, by monitoring the amplitude of PE signal as a function of the cavity length (L), let us directly determine the thermal diffusivity of a sample.

\section{EXPERIMENTAL DETAILS}

\subsection{Synthesis of Ag/clay nanofluids}

$\mathrm{AgNO}_{3}$ (99.98\%, Merck, Darmstadt, Germany) was used as the silver precursor, MMT clay powder (Kunipia-F, Tohoku, Japan) was used as the solid support for the Ag NPs, and $\mathrm{NaBH}_{4}(98.5 \%$, Sigma-Aldrich, St. Louis, MO, USA) was used as the reducing agent. All aqueous solutions were prepared in double-distilled water. We prepared the Ag/clay nanofluid by chemical reduction, as described in our earlier work [5]. The samples contained 1, 2, or $5 \mathrm{~g}$ of $\mathrm{Ag}$ and $100 \mathrm{~g}$ of MMT clay. Constant amounts of clay were suspended in different volumes of $1 \times 10^{-3} \mathrm{M} \mathrm{AgNO}_{3}$ aqueous solutions. These solutions were stirred for $24 \mathrm{~h}$ at room temperature. Freshly prepared $\mathrm{NaBH}_{4}\left(4 \times 10^{2} \mathrm{M}\right)$ solution was then added to the $\mathrm{AgNO}_{3}$ to attain a constant molar ratio of $1: 4 \mathrm{AgNO}_{3} / \mathrm{NaBH}_{4}$. These solutions were further stirred for $1 \mathrm{~h}$. The suspensions were then centrifuged, washed with double-distilled water to remove excess $\mathrm{NaBH}_{4}$, and dried under vacuum overnight. The powders were then suspended again in distilled water and dispersed with an ultrasonicator for $0.5-1 \mathrm{~h}$ to ensure proper mixing of the Ag NPs into the solutions. We found that all concentrations of Ag NPs were well dispersed in the fluids and had formed stable suspensions. In order to investigation the effect of agglomeration over time on thermal diffusivity of nanofluid, the Ag/clay $5 \mathrm{wt} \%$ nanofluid, after one month storage at room temperature, was stirred and then subjected to probe sonication at 1 hour to obtain uniform dispersions. The NPs in the nanofluids were characterized by using transmission electron microscopy (TEM; Hitachi H-7100, Tokyo, Japan) and ultraviolet-visible absorption spectroscopy (PerkinElmer, Waltham, MS, USA). Particle-size distributions were determined by using UTHSCSA Image Tool 3.0 software.

\subsection{Thermal Characterization}

Our experimental method has been reported in detail elsewhere $[15,16]$. Briefly, as shown in Figure 1, a 200-mW CW diode-pumped solid-state laser [MGL 150(10)] was modulated to $6.8 \mathrm{~Hz}$ by using an optical chopper (SR540); this frequency is considered optimal in our system for thermally thick samples, both for detection and SNR at a satisfactory signal amplitude. The beam was focused onto an inlet with a diameter of $2.25 \mathrm{~mm}$ in a single-core polymer fiber (RS 368-047) with a core diameter of $1 \mathrm{~mm}$. The modulated laser beam was channeled through the fiber to illuminate a very thin silvermetallized layer that coated the outlet fiber tip (1 mm thickness), to act as thermal wave generator. Before coating the end surface with silver conductive paint, it was coated with 


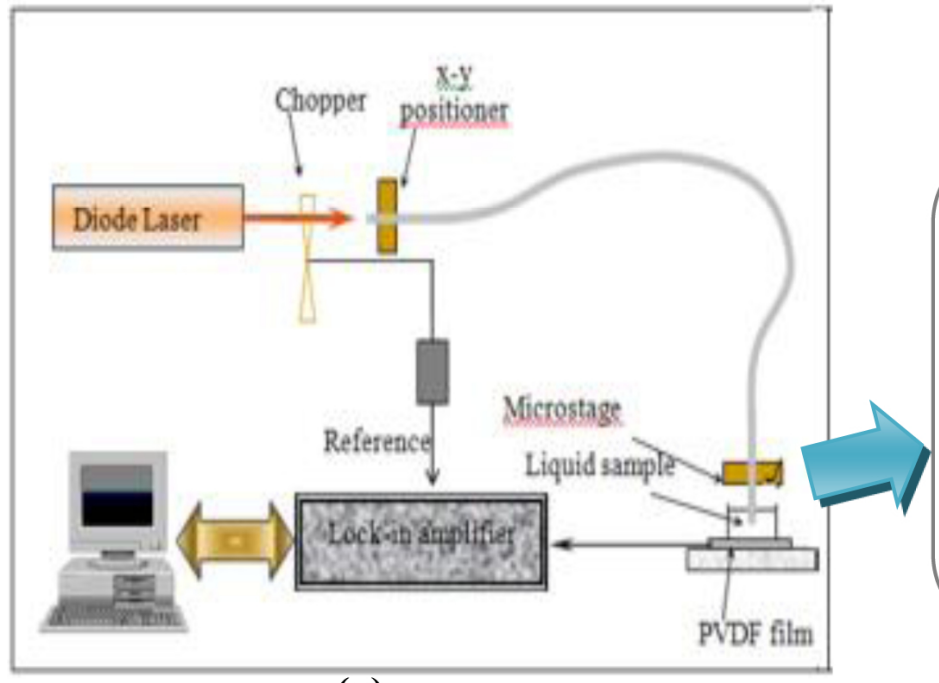

(a)

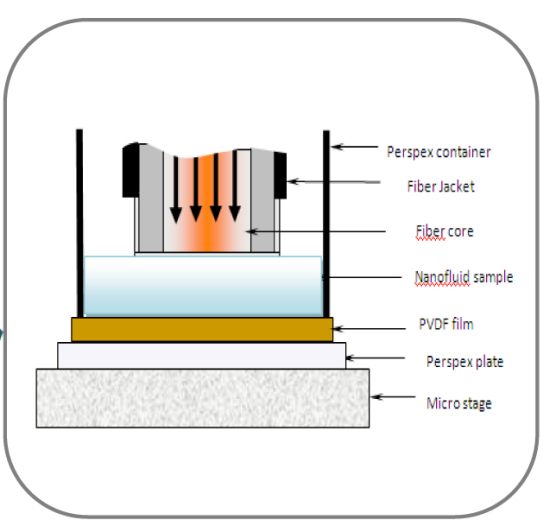

(b)

FIG. 1 (a) Schematic diagram of OF-TWC technique and (b) optical fiber and PE cell.

a very thin layer of matte black paint and polished to reasonable flatness so it could efficiently convert light to heat. The detection cell consisted of a sensitive PE detector made from a polyvinylidene fluoride (PVDF) (MSIDT1-028K/L, thickness of $52 \mu \mathrm{m}$ ). Used as a sample container, a plastic ring (diameter of $1 \mathrm{~cm}$ ) was glued to the top surface of the cell. The small volume of the nanofluid $(0.2 \mathrm{~mL})$ filled the inner side of the ring to a depth of $\sim 1 \mathrm{~mm}$. The cavity-length scan was performed by varying the distance $L$ between the fiber tip and the PE detector in resolution steps of $10 \mu \mathrm{m}$. The generated PE signal, measured by the PE film detector, was analyzed by using a lock-in amplifier to obtain the PE amplitude and phase signals. The noise level of the setup was $\sim 75 \mu \mathrm{V}$.

\section{RESULTS AND DISCUSSION}

\subsection{Sample Preparation}

In this research, MMT clay was appropriate as a stabilizer and organic polymer media for reducing the $\mathrm{AgNO}_{3}$ using $\mathrm{NaBH}_{4}$ as the reducing agent. The Ag NPs formed in the interlayer spaces of the MMT layers, separating the particles and preventing agglomeration. In the Ag NP/clay nanofluid, a very stable suspension in colloid form according to Eq. (3) as follows [19]:

$$
\begin{aligned}
\mathrm{Ag}^{+} / \text {clay }+\mathrm{BH}_{4^{-}} & +3 \mathrm{H}_{2} \mathrm{O} \\
& \rightarrow \mathrm{Ag}^{0} / \text { clay }+\mathrm{B}(\mathrm{OH})_{3}+3.5 \mathrm{H}_{2}
\end{aligned}
$$

Figure 2(a)-(c) shows TEM images of the Ag NPs, revealing average particle sizes of $4.19,6.74$, and $9.17 \mathrm{~nm}$ at concentrations of 1, 2, and $5 \mathrm{wt} \%$, respectively. The Ag NPs were spherical, but the MMT clay had nanofibrous shapes that only could be observed with high-resolution TEM. The nanofibrous structure of the MMT forms a nanocomposite and separates the Ag NPs, making the Ag/clay a suitable structure to support the Ag particles while controlling their size [3]-[5], $[20,21]$. Increasing the Ag NP concentration increased their average size. We attribute this behavior to the action of the

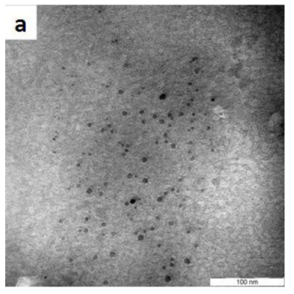

Mean Diameter: $4.19 \mathrm{~nm}$ Standard Deviation: $1.37 \mathrm{~nm}$

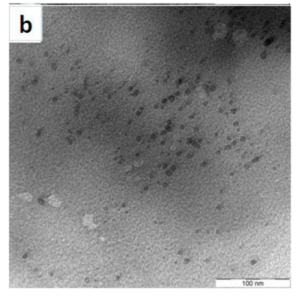

Mean Diameter: $6.74 \mathrm{~nm}$ Standard Deviation: $1.76 \mathrm{~nm}$

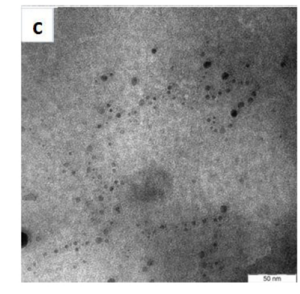

Mean Diameter: $9.17 \mathrm{~nm}$ Standard Deviation: $2.49 \mathrm{~nm}$
FIG. 2 TEM images and the corresponding particle size distributions of prepared Ag NPS in Clay suspension at different $\mathrm{AgNO}_{3}$ concentrations. (a) $1 \mathrm{wt} \%$, (b) $2 \mathrm{wt} \%$, (c) and 5 wt \%.

clay as an effective protective reagent: as the concentration increased, the basal plane of the clay layers could adsorb so many NPs, leaving the rest free in the bulk solution to aggregate and increase the average particle size [20]. Figure 3 shows the ultraviolet-visible absorption spectra of $\mathrm{Ag} /$ clay nanofluids at different concentrations. The solution without $\mathrm{NaBH}_{4}$ (Figure 3(a)) exhibited no characteristic SPR band absorption of the Ag NPs. In contrast, the solution with $\mathrm{NaBH}_{4}$ (figure $3 b$ ) exhibited the characteristic SPR band for the Ag NPs, at $\sim 420 \mathrm{~nm}$, confirming their formation. Increases in the concentration of $\mathrm{AgNO}_{3}$ corresponded to increases in the peak intensity absorption $\left(\lambda_{\max }\right)$, Figure $\left.3(\mathrm{~b})-(\mathrm{d})\right)$ [22].

\subsection{Experimental results:}

\subsubsection{Effect of concentration}

We assessed and calibrated the accuracy of the experimental system by measuring the thermal diffusivity of distilled water, fitting the PE signal of the logarithmic amplitude (Eq. (2)) versus the cavity length. The average thermal diffusivity of water, $1.439 \pm 0.019 \times 10^{-3} \mathrm{~cm}^{2} / \mathrm{s}$, differed by $<1 \%$ from literature values [22]. Figure 4(a) shows the amplitude of PE signal of clay with and without Ag NPs as a function of the relative cavity length. As shown in figure the PE signal in the sample attenu- 
ated rapidly to zero by increasing the cavity length. It is clear from the figure that at high cavity length the anomalous signal is very small, so the factor SNR is too small. Therefore, the PE signals were fitted with only in the useful cavity length [24], as can be seen in Figure 4(b). The curves of ln amplitude of PE signal vs cavity length of clay with and without Ag NPs are linear as shown in Figure 4(b). As a result, in a given intracavity medium, the PE signal of sample is related to its thermal diffusion length, samples with stronger PE signal had bigger thermal diffusivity. Then the accuracy of PE signal for sample with higher thermal diffusivity is stranger. Therefore, it is possible to have higher signal to noise ratio and the measurement can be carried out in high and stable PE signal. Such results are in accordance with the Photoacoustic method [23]. The less steep of the PE signal curve for Ag/clay nanofluid indicate that it has higher thermal diffusivity [24, 25] than the pure clay suspension, as shown in Figure 4(a). The thermal diffusivity average values can be calculated from the slope of the linear part of the logarithmic amplitude of PE signal curves using (Eq (2)). The slope of the PE signal curves will yield the thermal diffusivity of the nanofluids. This allowed for maximum sensitivity in the PE signal with respect to the change in $\mathrm{Ag} / \mathrm{Clay}$ nanofluids. Figure 5 shows that the thermal diffusivity ratio $\left(\alpha_{\text {nanofluid }} / \alpha_{\text {basefluid }}\right)$ of the nanofluids increased with increasing nanoparticle concentration in the clay suspension. Table I summarizes the slopes of the PE signal $[\ln (V)]$, and the resulting thermal diffusivity values for colloidal Ag/clay nanofluids with different NPs concentration.

The results show that the thermal diffusivity of Ag/Clay nanofluids are higher than the thermal diffusivity of the clay suspension. The reason for such anomalously high thermal diffusivity was the small size of nanoparticles that allows for more heat transfer between particle and base fluid and

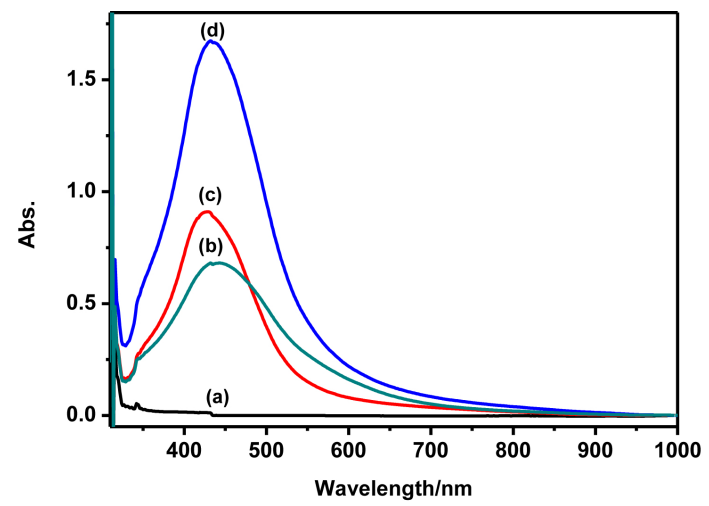

FIG. 3 UV-vis absorption spectra of prepared Ag NPs in Clay suspension at different $\mathrm{AgNO}_{3}$ concentrations, (a) $\mathrm{AgNO}_{3} /$ Clay (b) Ag/Clay $1 \%$ (c) Ag/Clay $2.0 \%$ (d) Ag/Clay $5.0 \%$. high thermal conductivity of particle materials [26]. The results show possible enhancement of thermal diffusivity ratio ( $\alpha$ sample/ $\alpha$ base fluid) of 1.08 for $1 w t \%, 1.11$ for $2 \mathrm{wt}$ $\%$,and 1.27 for $5 \mathrm{wt} \%$ NPs concentration. As can be seen in result, the nanofluids 1 and $2 \mathrm{wt} \%$ concentration yields no discernable changes in the ratio of thermal diffusivity, while, the $5 \mathrm{wt} \%$ concentration yields a larger enhancement in thermal diffusivity of nanofluid. Higher concentration in $5 \mathrm{wt} \%$ yield much faster Brownian diffusion and larger enhancement in thermal diffusivity [27]. Moreover, the increasing volume of the nanoparticles paired with the decrease in the specific heat of the nanofluids; consequently, the thermal diffusivity of the nanofluid increases [26]. As the nanoparticle concentration in the clay suspension increases, the quantity and size
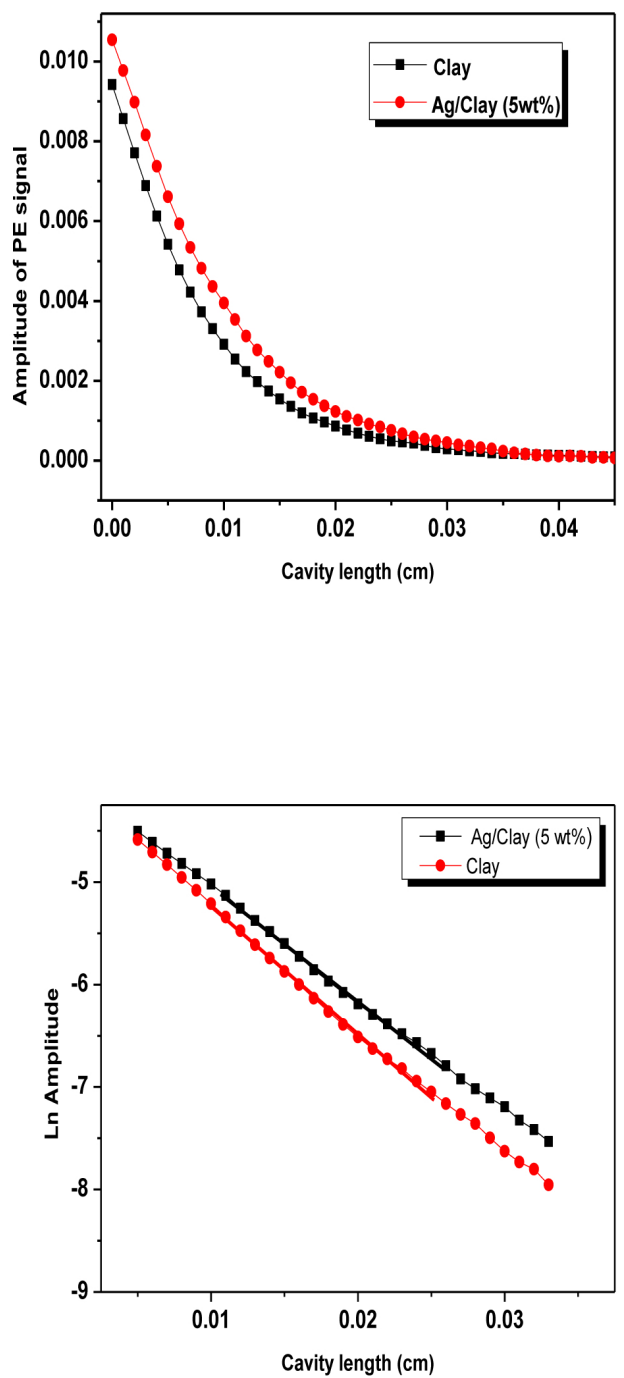

FIG. 4 Measured (a) amplitude (b) logarithmic amplitude of the PE signal in clay with and without Ag NPS as a function of the relative cavity length.

\begin{tabular}{|c|c|c|c|}
\hline wt $\mathbf{( \% )}$ & $\mathbf{A}_{\text {average }}$ & $\left.\alpha \mathbf{( 1 0}^{-\mathbf{3}} \mathbf{c m}^{\mathbf{2}} / \mathbf{s}\right)$ & $\alpha_{\text {nanofluid }} / \alpha_{\text {basefluid }}$ \\
\hline $\mathbf{0}$ & $124.92 \pm 0.98$ & $1.403 \pm 0.021$ & - \\
\hline $\mathbf{1}$ & $119.89 \pm 0.91$ & $1.524 \pm 0.023$ & 1.08 \\
\hline $\mathbf{2}$ & $118.46 \pm 1.13$ & $1.559 \pm 0.029$ & 1.11 \\
\hline $\mathbf{5}$ & $110.69 \pm 0.68$ & $1.789 \pm 0.022$ & 1.27 \\
\hline
\end{tabular}


of the nanoparticles increase, decreasing the specific heat of the nanofluid and increasing its thermal diffusivity. Similar results have been reported for thermal diffusivity measurements of metal nanofluids using the hot-wire method [11][13]. Additionally, as the NP concentration increases, the effective surface area in the fluid can increase, increasing the Brownian motion of the particles [26], which enables greater aggregation over time.

\subsubsection{Effect of Agglomeration time}

To study the effect of agglomeration time on nanofluids, first, UV-Visible spectroscopy was used to monitor how the nanoparticles change over time. Figure 6 shows the absorption spectra of $5 \mathrm{wt} \% \mathrm{Ag} /$ clay nanofluids for (a) before and (b) after one month storage at room temperature. As can be seen in Figure, there are a red shift of the absorption peak from 420 to $427 \mathrm{~nm}$ and the intensity of pick was decreased over time. This means the agglomeration of AgNPs has occurred, and the changed in dispersion intensity and the peak broadening shows the Ag/clay nanofluid became unstable over time [22]. Figure 7(a)-(b)) shows TEM image of the particle size after one month storage, from figure the clearly show the Ag NPs are large and not uniform in size with the mean particle diameter of $16.6 \mathrm{~nm}$ and standard deviation of $6.2 \mathrm{~nm}$.

It is defined as agglomeration effect in the thermal diffusivity gradually decreases only $7.3 \%$ after this time. This results show the Ag/Clay nanofluid has reasonably good stability behaviour and therefore the agglomeration time was in longer time. The clay suspension can help to minimize aggregation

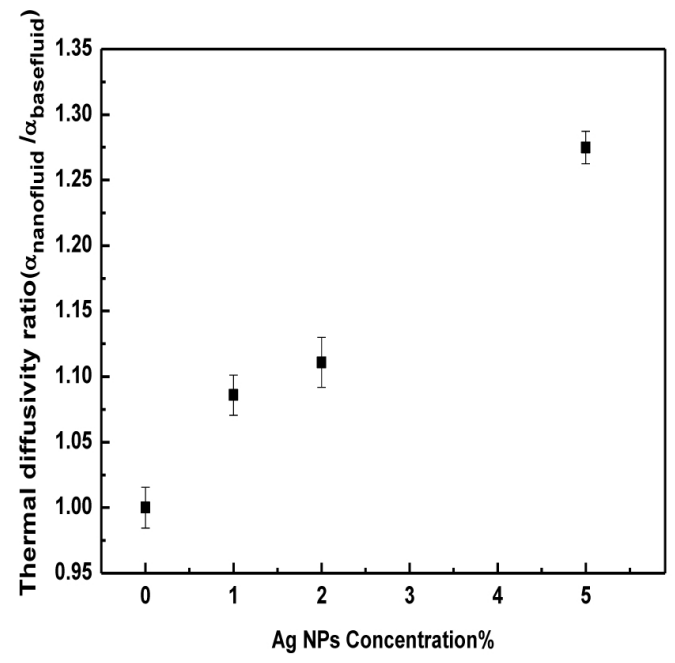

FIG. 5 Thermal diffusivity ratio ( $\alpha_{\text {nanofluid }} / \alpha_{\text {basefluid }}$ ) of Ag/Clay nanofluids at different $\mathrm{AgNO}_{3}$ concentration $0,1,2$ and $5 \mathrm{wt} \%$. of nanoparticles and restrict particle growth and can be good candidates as a base fluid for heat transfer applications. However, even for the stable nanofluids, there is a possibility that the agglomeration of nanoparticles over time decrease the thermal diffusivity of nanofluids [28]. It is worthwhile to note here that the observed thermal diffusivities are found to be in good agreement with the earlier reported results [11]-[13], $[23,27]$. This implies that the OF-TWC technique is very simple, less time consuming and powerful method for the thermal and optical characterization of nanofluids using laser.

\section{CONCLUSION}

We investigated the effects of concentration and agglomeration of nanoparticles on the thermal diffusivity of colloidal $\mathrm{Ag} /$ clay nanofluids prepared by using chemical reduction.

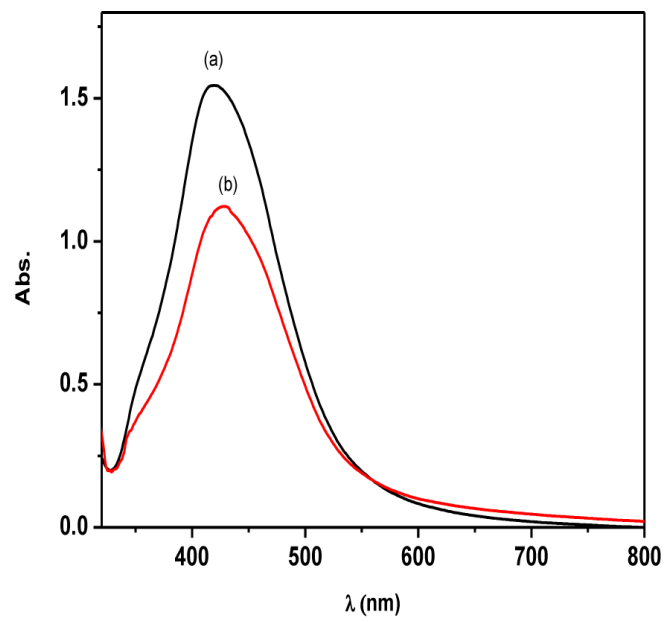

FIG. 6 The corresponding UV-Vis spectra of the $5 \mathrm{wt} \% \mathrm{Ag} / \mathrm{clay}$ nanofluid, (a) initial fresh and (b) storage over one month; absorption peak was red-shifted and broadened.

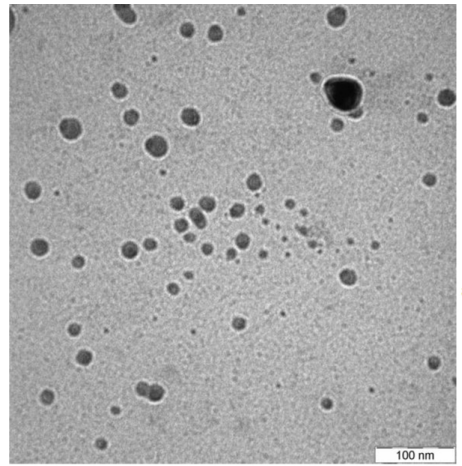

(a)

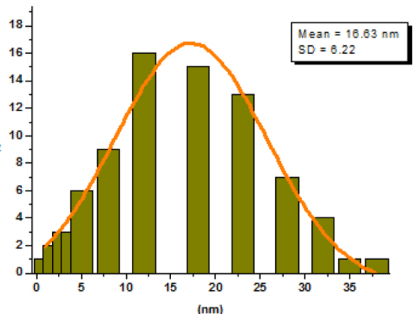

(b)
FIG. 7 (a) TEM image of Ag NPs and (b) histogram distribution of the particle size.

\begin{tabular}{|c|c|c|c|}
\hline Time hours & $\mathbf{A}_{\text {average }}$ & $\left.\alpha \mathbf{( 1 0}^{-\mathbf{3}} \mathbf{~} \mathbf{~}^{\mathbf{2}} / \mathbf{s}\right)$ & $\alpha_{\text {nanofluid }} / \alpha_{\text {basefluid }}$ \\
\hline 0 & 117.20 .8 & $1.594 \pm 0.011$ & 1.126 \\
\hline $\mathbf{1}$ & $118.0 \pm 0.6$ & $1.571 \pm 0.008$ & 1.110 \\
\hline $\mathbf{2}$ & $120.4 \pm 0.8$ & $1.509 \pm 0.010$ & 1.066 \\
\hline $\mathbf{3}$ & $122.6 \pm 0.6$ & $1.456 \pm 0.007$ & 1.029 \\
\hline
\end{tabular}


The interlamellar spaces of the clay offered high surface area to support the Ag NPs, and the Ag/clay structure suitably prevented agglomeration of the Ag NPs and controlled the particle size. The thermal diffusivity ratio increased with the concentration of $\mathrm{AgNO}_{3}$ over 1-5 wt.\%. The agglomeration of NPs over time results in not only the size of the cluster and particles but also the decrease of thermal properties as well as the thermal diffusivity of nanofluids. These results revealed similar behaviors in how the NP concentration enhanced the thermal diffusivity ratio compared with measurements using previously reported techniques. Thus, the high sensitivity of OF-TWC technique raises the potential to be applied to measuring the optical and thermal properties of nanofluids, while, and only required small amounts of sample $(0.2 \mathrm{~mL})$ per measurement with short enough experiment time.

\section{ACKN OWLEDGEMENT}

The authors are grateful to the Ministry of Science, Technology and Innovation for supporting this work under the Research University Grant Scheme No. 05-02-12-1878RU. The financial support from the Universiti Kebangsaan Malaysia (UKM) with project code DPP-2014-055 is acknowledged. We also acknowledge the financial assistance of the government of Malaysia.

\section{References}

[1] J. A. Eastman, U. S. Choi, S. Li, G. Soyez, L. J. Thompson, and R. J. DiMelfi, "Novel thermal properties of nanostructured materials," J. Metastab. Nanocryst. 2, 629-634 (1999).

[2] R. G. Fuentes, J. A. P. Rojas, J. L. J. Pérez, and J. F. S. Ramirez, "Study of thermal diffusivity of nanofluids with bimetallic NPs with $\mathrm{Au}$ (core)/Ag (shell) structure," Apsusc. 255(3), 781-783 (2008).

[3] H. Huang, and Y. Yang, "Preparation of silver nanoparticles in inorganic clay suspensions," Compos. Sci. Technol. 68, 2948-2953 (2008).

[4] R. A. Patakfalvi, A. Oszka, and I. Dekany, "Synthesis and characterization of silver nanoparticles/kaolinite composites," Colloid. Surface. A 220, 45-54(2003).

[5] M. B. Ahmad, K. Shameli, M. Darroudi, and W. Yunus, "Synthesis and Characterization of Silver/Clay Nanocomposites by Chemical Reduction Method," American Journal of Applied Sciences, 6(11), 1909-1914 (2009).

[6] R. Prasher, W. Evans, P. Meakin, J. Fish, P. Phelan, and P. Keblinski, "Effect of aggregation on thermal conduction in colloidal nanofluids," Appl. Phys. Lett. 89(14), 143119 (2006).

[7] S. A. Putnam, D. G. Cahill, and P. V. Braun, "Thermal conductivity of nanoparticle suspensions," J. Appl. Phys. 99, 084308 (2006).

[8] P. Keblinski, J. A. Eastman, and D. G. Cahill “Nanofluids for thermal transport," Mater. Today 8(6), 36-44 (2005).

[9] S. M. S. Murshed, K. C. Leong, and C. Yang, "Enhanced thermal conductivity of $\mathrm{TiO}_{2}$ - water based nanofluids," Int. J. Therm. Sci. 44, 367-373 (2005).

[10] C. Kleinstreuer, and Y. Feng, "Experimental and theoretical studies of nanofluid thermal conductivity enhancement: a review," Nanoscale Res. Lett. 6, 229 (2011).
[11] S. M. S. Murshed, K. C. Leong, and C. Yang, "Determination of the effective thermal diffusivity of nanofluids by the double hot-wire technique," J. Phys. D: Appl. Phys. 39, 5316-5322 (2006).

[12] R. Zamiri, B. Z. Azmi, E. Shahriari, and K. Naghavi, "Thermal diffusivity measurement of silver nanofluid by thermal lens technique," J. Laser Appl. 23, 042002 (2011).

[13] X. Zhang, H. Gu, and M. Fujii, "Effective thermal conductivity and thermal diffusivity of nanofluids containing spherical and cylindrical nanoparticles," J. Appl. Phys. 100, 044325 (2006).

[14] J. Shen, and A. Mandelis, "Thermal-wave resonator cavity," Rev. Sci. Instrum. 66, 4999-5005 (1995).

[15] B. Z. Azmi, M. Noroozi, Z. Rizwan, Z. A. Sulaiman, Z. A. Wahab, and M. M. Moksin, "Simple TWRC technique by using optical fiber," Infrared Phys. Techn. 51(3), 270-275 (2008).

[16] M. Noroozi, B. Z. Azmi, and M. M. Moksin, "The reliability of optical fiber-TWRC technique in liquids thermal diffusivity measurement," Infrared Phys. Techn. 53(3), 193-196 (2010).

[17] A. Matvienko, and A. Mandelis, "Quantitative one-dimensional thermal-wave cavity measurements of fluid thermophysical properties through equivalence studies with three- dimensional geometries," Rev. Sci. Instum. 77, 1-9 (2006).

[18] J. Philip, and M. R. Nisha, "Thermal diffusion in dilute nanofluids investigated by photothermal interferometry," J. Phys.: Conf. Ser. 214, 012035 (2010).

[19] K. C. Song, M. S. Lee, T. S. Park, and B. S. Lee, "Preparation of colloidal silver nanoparticles by chemical reduction method," Korean J. Chem. Eng. 26, 153-155 (2009).

[20] I. Dekany, and R. Patakfalvi, "Synthesis and intercalation of silver nanoparticles in kaolinite/DMSO complexes," Appl. Clay Sci. 25, 149-159 (2004).

[21] M. D. Fan, P. Yuan, T. H. Chen, H. P. He, and A. H. Yuan, "Synthesis, characterization and size control of zerovalent iron nanoparticles anchored on montmorillonite," Chin. Sci. Bull. 55(11), 1092-1099 (2010).

[22] R. Desai, V. Mankad, S. K. Gupta, and P. K. Jha, "Size Distribution of Silver Nanoparticles: UV-Visible Spectroscopic Assessment," Nanoscience and Nanotechnology Letters 4, 30-34 (2012).

[23] J. A. Balderas-Lopez, "Thermal effusivity measurements for liquids: A self-consistent photoacoustic methodology," Rev. Sci. Instrum. 78, $064901-064904$ (2007).

[24] B. Z. Azmi, L. T. Sing, E. B. Saion, and Z. A. Wahab, "Thermal wave interferometry of gas-liquid using optical fibre thermal wave resonator cavity technique," J. Sci. Technol. 14, 33-40 (2006).

[25] G. Pan, and A. Mandelis, "Measurements of the thermodynamic equation of state via the pressure dependence of thermophysical properties of air by a thermal-wave resonant cavity," Rev. Sci. Instrum. 69, 2918-2923 (1998).

[26] G. A. López-Muñoz, and J. A. Balderas-López, "Thermal diffusivity measurement for urchin-like gold nanofluids with different solvents, sizes and concentrations/shapes," Nanoscale Res. Lett. 7(1), 667 (2012).

[27] C. H. Chon, and K. D. Kihm, "Thermal conductivity enhancement of nanofluids by Brownian motion," J. Heat Transf. 127, 810 (2005).

[28] R. Prasher, P. E. Phelan, and P. Bhattacharya, "Effect of aggregation kinetics on the thermal conductivity of nanoscale colloidal solutions (nanofluid)," Nano Lett. 6(7), 1529-1534 (2006). 